\title{
Using spectroscopic analysis of unclassified supergiants to investigate massive stars in transition ${ }^{\star}$
}

\section{The superluminous HD 327083}

\author{
M. A. D. Machado and F. X. de Araújo \\ Observatório Nacional-MCT, Rua José Cristino, 77, CEP 20921-400, São Cristóvão, Rio de Janeiro-RJ, Brazil \\ Received 16 January 2003 / Accepted 7 July 2003

\begin{abstract}
New optical high-resolution $(R \approx 50000)$ spectra of the star HD 327083 are reported in this paper. The Balmer and Fe II lines show P Cygni profiles while He I lines are all in pure absorption, resembling the spectra of the Luminous Blue Variable HR Carinae. However, the blue absorption components of the Balmer lines are more pronounced and the Fe II lines are less numerous and less intense in HD 327083. A spectroscopic analysis of the Balmer lines $(\mathrm{H} \alpha, \mathrm{H} \beta, \mathrm{H} \gamma$ and $\mathrm{H} \delta)$ with a non-LTE wind model has been performed. The derived stellar parameters for HD 327083 are: $\log \left(L_{\star} / L_{\odot}\right)=6.0, T_{\star}=11500 \mathrm{~K}$, $\dot{M}=8.0 \times 10^{-5} M_{\odot} / \mathrm{yr}$ and $\mathrm{He} / \mathrm{H}=0.2$ (by number). From these results we concluded that HD 327083 is a superluminous object in an evolved phase of an evolutive track of $M_{\text {ZAMS }} \sim 60 M_{\odot}$. It is likely to be about to enter in a Luminous Blue Variable phase.
\end{abstract}

Key words. stars: emission-line, Be - stars: variables: general - stars: individual: HD 327083

\section{Introduction}

The upper part of the HR Diagram is populated by massive stars with luminosities between $10^{5}$ and $10^{6} \mathrm{~L}_{\odot}$. Their initial masses on the Zero Age Main Sequence are greater than 25-30 $M_{\odot}$. These stars present an extense temperature range: $40000 \mathrm{~K} \geq T_{\star} \geq 8000 \mathrm{~K}$. The upper limit corresponds to the temperatures of the stars with initial mass around 80-120 $M_{\odot}$. The lower limit is defined by the Humphreys-Davidson (1979) instability limit, related to the observed lack of cool highluminosity stars. They are distributed in spectral classes that roughly correspond to distinct phases in their lives. For each initial mass, they evolve at almost constant luminosity and temperature ranges can be assigned to different evolutionary phases. However, the most characteristic parameter of massive stars is the mass loss rate of the stellar winds that are observed in all phases and systematically peels off the external layers showing the nucleosynthetized material. Nowadays the most accepted scenarium for the evolution of very massive stars with $M_{\text {ZAMS }} \geq 50-60 M_{\odot}$ consists basically in the following sequence: $\mathrm{O}$ type-star (hydrogen core-burning stars at main sequence) $\rightarrow$ Of (high mass loss from steady stellar winds) $\rightarrow$ LBV (photometric and spectroscopicaly variable stars undergoing intense episodic mass ejection events) $\rightarrow$ WNL (nitrogen Wolf-Rayet stars with some hydrogen) $\rightarrow$ WNE

Send offprint requests to: M. A. D. Machado, e-mail: dora@on.br

* Based on observations made with the $1.52 \mathrm{~m}$ telescope at the European Southern Observatory (La Silla, Chile) under the agreement with the Observatório Nacional, Brazil. (hydrogen-poor or absent nitrogen Wolf-Rayet stars) $\rightarrow$ WC (carbon Wolf-Rayet stars) $\rightarrow$ (WO) $\rightarrow$ SN. This sequence was proposed some years ago by Geneve's group (Maeder \& Meynet 1987; Schaller et al. 1992). The theory has been greatly improved in recent years (Maeder \& Meynet 2000) but the overall picture for slow rotating stars with normal metalicities remains the same. On the other hand the localization of the luminous blue variable (LBV) phenomenon is controversial. According to Langer et al. (1994) it may occur between the WNL and WNE phases. Nevertheless, all the models indicate a sequence of continuous enrichment of the stellar wind with the products of nuclear burning.

The transitions undergone by massive stars during the intermediate phases and the possible connections between these stages are still not clear. A major problem is the small number of stars in each transitional phase. The identification of the transition phases is hampered by changes on spectral features due to different mechanisms. A good example is given by the Ofpe/WN9 stars. This term was introduced by Walborn (1982) to define objects that could not be classified only as Of nor as WNL stars. For instance R127 (a supergiant in the Large Magellanic Cloud) which was classified as an Ofpe/WN9 star until it presents photometric and spectroscopic characteristics of a LBV, as reported by Stahl et al. (1983). On the other hand, the well known LBV AG Carinae at visual minimum shows observational features of an Ofpe/WN star (Stahl 1987). Nota et al. (1996) used optical spectroscopic data to analyse the morphology of some Ofpe/WN stars and related objects. According to them, these stars constitute a heterogeneous 
group with characteristics varying from the Of to the $\mathrm{B}[\mathrm{e}]$ stars. In addition, they pointed out that the spectra of two Ofpe/WN9 stars show nebular emission lines formed in expanding shells associated with the central object. This fact suggests a connection of Ofpe/WN9 stars with LBVs, since LBVs are generally surrounded by circumstellar nebulae produced by outburts in their evolutive paths. These results are supported by Morris et al. (1996) that analyzed the morphology of IR spectra of several Ofpe/WN and related stars. Nota et al. (1996) and Morris et al. (1996) also discuss evidences of spectral morphological connections among B[e] Supergiants and LBVs. Such a connection was first suggested by Schulte-Ladbeck et al. (1993) which used a circumstellar disk, characteristic of $\mathrm{B}[\mathrm{e}]$ Supergiants, to explain the polarization in the wind of R127. All this evidence, as well as many others in the literature, show how complex it can be to identify and classify a massive star in transition.

A powerful method to derive physical parameters and estimate the evolutionary phase of an object is given by the fitting of theoretical line profiles obtained with adequate atmospheric codes and comparison with evolutive tracks. Schmutz et al. (1991) performed a spectral analysis of the Ofpe/WN9 star HD 269227 (R84). They found that it has lost half of its initial mass. The authors also suggested that R84 is probably in a post-red supergiant phase and that it must have undergone violent mass loss in that phase. In the same work the authors concluded that at temperatures close to $30000 \mathrm{~K}$ the He II $\lambda 4686$ line is very sensitive to temperature and a tight domain of $\pm 1000 \mathrm{~K}$ could be derived. Smith et al. (1994) presented a spectroscopic study of the supergiant He 3-519 and of AG Carinae at visual minimum, using the atmospheric model by Hillier (1987, 1990). They fitted the H $\beta$, H I $\lambda 4471$ and He II $\lambda 4686$ line profiles. According to them the temperature values $(T=27000 \mathrm{~K}$ for He $3-519$ and $T=25000 \mathrm{~K}$ for AG Car) and the low hydrogen content found for both objects are consistent with a WN11 type. From these results, Smith et al. (1994) have proposed that LBVs show spectral characteristics typical of late spectral types of the WN sequence. Recently Stahl et al. (2001) have published a spectroscopic monitoring of AG Carinae during a time interval that corresponds to an almost complete S Dor cycle. They have chosen some phases to fit the $\mathrm{H} \alpha$ profile in order to investigate its mass loss history. The computation was made with a code described in Schmutz (1997). The authors showed that at the beginning of the cycle there is a slight increase of mass loss rate that returns to the minimum phase value afterwards. Accordingly, Stahl et al. (2001) concluded that they have no evidence to associate the origin of the $\mathrm{S}$ Dor cycle with changes in the mass loss rate, as proposed by Davidson (1987).

Some more specific aspects have been also analysed using results produced by atmospheric models. Pasquali et al. (1997) used an atmospheric code to adjust the lines He II $\lambda$ 4686, H $\alpha$ and $\mathrm{He} \mathrm{I} 1.083 \mu \mathrm{m}$ in order to derive the stellar and wind parameters of HD 269445 (R99). The input parameters that fitted He II $\lambda 4686$ and He I $1.083 \mu \mathrm{m}$ absorptions do not reproduced well $\mathrm{H} \alpha$ and He I $1.083 \mu \mathrm{m}$ emissions. They interpreted the discrepancies as the effects of a non-spherical wind which has a hot, fast polar component and a cold, slow equatorial component. Hillier et al. (1998) investigated in detail the optical and near-infrared spectra of the supergiant HD 316285. The observed profiles of the Balmer lines were well reproduced while the Paschen and He I lines were not. The inability to reproduce all observed profiles must come from limitations of the model as inappropriate velocity law, neglecting of possible instabilities in the wind or from the rotation effects that were not considered. Regarding this last mechanism, HD 316285 exhibites evidences of a line effect in $\mathrm{H} \alpha$ that indicates the possibility of intrinsic polarization suggesting that rotation can be important. In spite of all uncertainties the $\mathrm{He} / \mathrm{H}$ ratio and high mass loss rate derived are indications of an evolved evolutionary phase of short duration. The association with the LBV phase is reinforced by the spectral similarity with $\mathrm{P}$ Cygni, presence of $\mathrm{CNO}$ processed material at its surface, spectral variability and the presence of a nebulae. Even the well studied LBV P Cygni was revisited in the light of atmospheric models. Najarro et al. (1997) investigated how line strengths, line shapes and the emergent energy distribution behave as functions of the fundamental stellar parameters. The fits of observed optical and nearinfrared $\mathrm{H}$ and He I profiles as well as the reproduction of continuum fluxes confirmed that P Cygni is highly evolved. More recently, Machado et al. (2002, hereafter Paper I) analysed the LBV HR Carinae, using the code developed by Machado (1998). From the fitting of Balmer lines we have estimated, among other parameters, a ratio $\frac{\mathrm{He}}{\mathrm{H}}=0.4$ by number and a temperature $T_{\star}=10000 \mathrm{~K}$. The comparison of this temperature with those obtained previously by Nota et al. (1997) allowed us to conclude that HR Car is moving in the $\mathrm{H}-\mathrm{R}$ diagram in a $\mathrm{S}$ Doradus-type cycle. All examples listed above show how the results obtained with the atmospheric codes can help to understand massive stars.

The poorly-studied object HD 327083 is another example of a peculiar star that deserves further spectroscopic analysis based on an adequate atmospheric model. The data available in the literature are not enough to define HD 327083 as a member of a class. This object belongs to the list of southern emission line stars of Carlson \& Henize (1979). McGregor et al. (1988) carried out infrared spectroscopic observations of some objects from that sample. They detected the first-overtone $\mathrm{CO}$ emission for six stars, including HD 327083. According to the authors this emission is formed in a circumstellar shell. Lopes et al. (1992) reported observations of a few short spectral regions of HD 327083 (among other luminous objects). They showed H I and Fe II $\lambda \lambda$ 4924, 5018, 5169 (m42) lines with P Cygni-type profiles, while the Fe II $\lambda 9997$ profile seemed to be in pure emission. The authors have suggested a B6 type. More recently, Thé et al. (1994) presented a new catalogue of (confirmed and candidate) Herbig AeBe stars. In this catalogue HD 327083 is cited among the extreme emission-line objects, which are not identified to belong to a certain group. However, they proposed the spectral type B1.5 that has been used by Valenti et al. (2000). The values $\log T_{\text {eff }} \geq 4.2$ and $\log \frac{L}{L_{\odot}} \sim 6.2$ were estimated by Sheikina et al. (2000) who analysed a sub-group of IRAS sources that show a rather steep decrease of the IR flux longward of 25 microns. This probably implies that these stars are surrounded by a warm dust formed recently.

HD 327083 drew our attention for its evolved condition when we analysed its $\mathrm{H} \alpha$ and $\mathrm{H} \beta$ lines (from 
medium-resolution spectra) using an atmospheric code to estimate its stellar parameters (Machado et al. 2001). Due to the reduced number of lines available we obtained the two models that best reproduced the observations. Each model is characterized by different values of temperatures and luminosities that place the object in a specific location in the HR diagram. Two alternatives - redward or blueward - are possible if only $T_{\star}$ and $L_{\star}$ are considered. He abundance may be invoked to explain each possibility. Model 1 has $A_{\mathrm{He}}=0.40$. This value is in the blueward track: the star would be coming back to the hotter region of the diagram. The parameters in this scenario point to a LBV quiescent (or post-LBV) phase. On the other hand model 2 has $A_{\mathrm{He}}=0.11$. This low value indicates a point in the redward track. Here the star is in a less evolved stage, coming to the LBV eruptive phase. In the present work we report new spectral data of HD 327083. Emphasis is given on the high-resolution data but a new low-resolution spectrum has also been obtained. The spectra are compared with those shown by HR Carinae and described in Paper I. Physical parameters are derived mainly from the fitting of $\mathrm{H} \alpha, \mathrm{H} \beta, \mathrm{H} \gamma$ and $\mathrm{H} \delta$ lines. The absence of He I emission helps to constrain the stellar temperature. Our results lead to a more precise interpretation of the evolutionary status of the object.

\section{The data}

\subsection{Observations, reduction and line identification}

Spectroscopic high-resolution data were observed with a Fiberfed Extended Range Optical Spectrograph (FEROS, see Kaufer et al. 1999), with the ESO $1.52 \mathrm{~m}$ telescope in La Silla (Chile) on April 19, 1999. FEROS is a bench-mounted Echelle spectrograph with the fibers located at the Cassegrain focus with a spectral resolving power of $R=48000$ corresponding to 2.2 pixels of $15 \mu \mathrm{m}$ and with a wavelength coverage from $\lambda 3600$ to $\lambda$ 9200. A completely automatic on-line reduction is available and it was adopted by us. The exposure time was $1200 \mathrm{~s}$, leading to a $S / N$ ratio in the continuum of approximately 34 in the $\lambda 5500$ region.

The low-resolution data were obtained using a Boller \& Chivens spectrograph at the Cassegrain focus on March 12, 1998. The observations were made with grating \#23 (600 $1 \mathrm{~mm}^{-1}$ ), providing a resolution of $4.6 \AA$ in the range $\lambda 3600-7400$. The Cassegrain spectra were reduced using standard IRAF tasks, such as bias subtraction, flat-field normalization, and wavelength calibration. For an exposure time of $10 \mathrm{~s}$ the $S / N$ ratio in the continuum is aproximately 114 in the $\lambda 5500$ region. The linearized and continuum-normalized spectrum of HD 327083 is shown in Fig. 1. Later on this paper we will compare HD 327083 features with those presented by HR Carinae. So, Fig. 1 also shows the low-resolution spectrum of this object, obtained with the same apparatus (see Paper I).

Line identification was carried out using the line lists provided by Moore (1945), Garcia-Lario et al. (1998), Landaberry et al. (2001), Fernandes et al. (2001) and Paper I. Table 1 summarizes the emission and absorption features identified in our spectrum of HD 327087. Column 1 gives the measured central wavelength of each feature. Column 2 lists our corresponding
Table 1. Observed emission and absorption lines.

\begin{tabular}{|c|c|c|}
\hline Wavelength $(\AA)$ & Identification & $W_{\lambda}(\AA)$ \\
\hline \multirow[t]{4}{*}{3970.0} & $\mathrm{Fe} \mathrm{II}(3), 69.38$ & 0.35 \\
\hline & Fe II $[8 \mathrm{~F}], 68.27$ & \\
\hline & Fe II[24F],68.66 & \\
\hline & $\mathrm{H} \epsilon$ & \\
\hline 4026.9 & $\operatorname{HeI}(18), 26.1+26.3$ & abs \\
\hline 4102.3 & $\mathrm{H} \delta$ & 0.81 \\
\hline 4120.0 & $\operatorname{HeI}(16), 20.8$ & abs \\
\hline 4128.6 & Si II(3),28.1 & abs \\
\hline 4131.4 & Si II(3),30.9 & abs \\
\hline 4144.5 & $\operatorname{HeI}(53), 43.7$ & abs \\
\hline \multirow[t]{2}{*}{4233.5} & $\mathrm{Fe} \mathrm{II}[21 \mathrm{~F}], 31.56$ & 0.14 \\
\hline & Fe II(27),33.167 & \\
\hline 4286.8 & Fe II[7F], 87.4 & 0.16 \\
\hline 4341.0 & $\mathrm{H} \gamma$ & 1.45 \\
\hline \multirow[t]{2}{*}{4352.0} & $\mathrm{Fe} \mathrm{II}(27), 51.8$ & 0.04 \\
\hline & Fe II[7F],52.8 & \\
\hline \multirow[t]{3}{*}{4358.8} & Fe II[6F],58.10 & 0.05 \\
\hline & $\mathrm{Fe}$ II[7F],59.34 & \\
\hline & Fe II[21F],58.37 & \\
\hline 4387.0 & HeI(51)87.93 & abs \\
\hline 4437.0 & $\operatorname{HeI}(50), 37.55$ & abs \\
\hline 4472.5 & $\operatorname{HeI}(14), 71.5$ & abs \\
\hline \multirow[t]{2}{*}{4508.6} & Fe II(38),08.28 & 0.07 \\
\hline & Fe II[6F],09.61 & \\
\hline \multirow[t]{3}{*}{4515.7} & Fe II(20), 15.19 & 0.07 \\
\hline & Fe II(37), 15.34 & \\
\hline & $\mathrm{Fe}$ II $[6 \mathrm{~F}], 14.90$ & \\
\hline 4520.6 & Fe II(37), 20.23 & 0.10 \\
\hline \multirow[t]{3}{*}{4584.2} & Fe II(37), 82.84 & 0.27 \\
\hline & $\mathrm{Fe} \mathrm{II}(38), 83.83$ & \\
\hline & Fe II(26),83.99 & \\
\hline
\end{tabular}

identifications for the feature, including ion, multiplet and rest central wavelength of the proposed transition. In some cases more than one transition may contribute to a given feature. Column 3 gives our estimate of equivalent width $\left(W_{\lambda}\right)$. For the emission lines we present the equivalent widths; in the case of P Cygni profiles only the equivalent width of the emission component was measured. If the equivalent width is very small $\left(W_{\lambda} \leq 0.01\right)$ but the transition is surely present, only the identification is given. The absorption lines are marked with abs.

\subsection{Description of the spectrum}

The optical spectrum of HD 327083 is marked by several P Cygni profiles from Balmer and single ionized iron lines. The $\mathrm{H} \alpha$ line is the more intense and show a strong central emission peak, a blueshifted absorption component and broad wings extended in the blue and red side of the line. These wings are attributed to electron scattering (Bernat \& Lambert 1978). The iron lines are permitted as well as forbidden. The strongest 
Table 1. continued.

\begin{tabular}{|c|c|c|}
\hline Wavelength $(\AA)$ & Identification & $W_{\lambda}(\AA)$ \\
\hline 4601.9 & N II(5),01.48 & 0.12 \\
\hline 4608.1 & N II(5),07.16 & 0.07 \\
\hline 4614.6 & N II(5),13.87 & 0.09 \\
\hline 4629.6 & Fe II(37),29.34 & 0.06 \\
\hline \multicolumn{3}{|l|}{$\mathrm{N}$ II(5),30.54 } \\
\hline 4642.1 & $\mathrm{~N}$ II $(5), 43,08$ & 0.07 \\
\hline \multirow[t]{2}{*}{4663.9} & Fe II(44),63.70 & 0.01 \\
\hline & $\mathrm{Fe} \mathrm{II}[4 \mathrm{~F}], 64.40$ & \\
\hline \multirow[t]{2}{*}{4665.2} & Fe II(37).66.80 & 0.05 \\
\hline & Fe II[4F],67.00 & \\
\hline 4714.2 & $\operatorname{HeI}(12), 13.1+13.4$ & abs \\
\hline 4814.1 & Fe II[20F],14.5 & 0.05 \\
\hline 4861.7 & $\mathrm{H} \beta$ & 3.9 \\
\hline 4889.5 & $\mathrm{Fe} \mathrm{II}[4 \mathrm{~F}], 89.63$ & 0.09 \\
\hline \multirow[t]{2}{*}{4921.5} & $\operatorname{HeI}(48), 21.93$ & abs \\
\hline & $\operatorname{HeI}(4), 15.675$ & abs \\
\hline 4924.2 & Fe II(42),23.92 & 0.40 \\
\hline 5007.9 & $\mathrm{Fe} \mathrm{II}[4 \mathrm{~F}], 06.52$ & 0.09 \\
\hline 5018.6 & Fe II(42),18.4 & 0.66 \\
\hline 5047.2 & $\operatorname{HeI}(47), 47.74$ & abs \\
\hline \multirow[t]{3}{*}{5158.1} & $\mathrm{Fe} \mathrm{II}[18 \mathrm{~F}], 58.00$ & 0.27 \\
\hline & Fe II[19F],58.81 & \\
\hline & Fe II(35),61.2 & \\
\hline 5169.6 & Fe II(42),69.03 & 0.48 \\
\hline 5183.4 & Fe II $[19 \mathrm{~F}], 84.8$ & 0.02 \\
\hline \multirow[t]{2}{*}{5197.8} & Fe II(49),97.569 & 0.06 \\
\hline & Fe II[35F],99.18 & \\
\hline 5235.1 & Fe II(40),34.62 & 0.18 \\
\hline 5276.4 & Fe II(49),75.99 & 0.10 \\
\hline 5282.5 & Fe II $[35 F], 83.10$ & 0.10 \\
\hline \multirow[t]{2}{*}{5316.9} & Fe II(49),16.61 & 0.27 \\
\hline & Fe II(48), 16.78 & \\
\hline 5326.4 & Fe II(49),25.5 & 0.21 \\
\hline 5336.9 & Fe II(48),37.7 & 0.03 \\
\hline 5363.3 & Fe II(48),62.86 & 0.08 \\
\hline 5656.4 & Fe II $[18 \mathrm{~F}], 56.31$ & 0.05 \\
\hline 5876.0 & HeI(11),75.6 & $\mathrm{abs}$ \\
\hline
\end{tabular}

features can be identified as being due to multiplet 42 at $\lambda$ $\lambda$ 4924, 5018 and 5169. The He I lines presented in our spectrum are all in pure absorption. No evidence is found for the presence of He II in the spectrum of HD 327083.

From the elements of CNO group we identify some multiplets of N II and O I. N II $\lambda$ 4601-43 is clearly in emission. O I $\lambda \lambda 7772-5$ shows a $\mathrm{P}$ Cygni profile, while $\mathrm{O}$ I $\lambda 8445$ is in emission. This last line can be produced by continuum fluorescence and Ly $\beta$ fluorescence. According to Garcia-Lario et al. (1998) if the origin of the emission is continuum fluorescence, the lines $\lambda 7002$ and $\lambda 7254$ would appear in emission but this does not occur. So this line is likely to be produced by Ly $\beta$ fluorescenc. The [N II] transitions at $\lambda \lambda 6548.1$ and 6583.6 [1F]
Table 1. continued.

\begin{tabular}{|c|c|c|}
\hline Wavelength $(\AA)$ & Identification & $W_{\lambda}(\AA)$ \\
\hline 5989.8 & & 0.13 \\
\hline 5890.0 & $\mathrm{NaI}(1), 89.95$ & abs \\
\hline 5895.9 & $\operatorname{NaI}(1), 96.0$ & abs \\
\hline 5991.8 & Fe II(46),91.4 & 0.07 \\
\hline 6045.4 & Fe II[a2G-a2I],44.10 & 0.01 \\
\hline 6082.5 & Fe II(46),84.1 & 0.04 \\
\hline 6148.3 & Fe II(74), 47.74 & 0.05 \\
\hline 6149.9 & Fe II(74),49.24 & 0.07 \\
\hline 6231.3 & $\mathrm{Fe}$ II $[39 \mathrm{~F}], 31.277$ & 0.02 \\
\hline 6238.6 & Fe II(74),38.42 & 0.03 \\
\hline 6248.3 & $\mathrm{Fe} \mathrm{II}(74), 47.56$ & 0.11 \\
\hline 6299.6 & O I $[1 \mathrm{~F}], 00.23$ & 0.18 \\
\hline 6300.0 & $\mathrm{O} \mathrm{I}, 00.2$ & 0.12 \\
\hline 6316.6 & Fe II(z4D0-c4D), 17.98 & 0.04 \\
\hline 6347.0 & Si II, 46.2 & abs \\
\hline 6363.0 & O I,63.0 & 0.07 \\
\hline 6371.0 & $\mathrm{Si}$ II, 70.7 & abs \\
\hline 6379.6 & N II,79.2 & abs \\
\hline 6383.3 & Fe II(z4D0-c4D),83.75 & 0.16 \\
\hline 6417.5 & $\mathrm{Fe} \mathrm{II}(74), 16.9$ & 0.05 \\
\hline 6457.8 & Fe II(74),56.37 & 0.10 \\
\hline 6506.0 & $\mathrm{Ne}, \mathrm{I}, 06.4$ & abs \\
\hline 6516.6 & Fe II(40), 16.05 & 0.18 \\
\hline 6547.2 & $\mathrm{~N}$ II[1F],48.1 & \\
\hline 6563.2 & $\mathrm{H} \alpha$ & 26.79 \\
\hline 6584.0 & $\mathrm{~N}$ II $[1 \mathrm{~F}], 83.6$ & 0.20 \\
\hline 6614.0 & Fe,I,13.6 & abs \\
\hline 6679.1 & $\operatorname{HeI}(46), 78.1$ & abs \\
\hline 7063.4 & $\operatorname{HeI}(10), 65.72$ & abs \\
\hline 7154.1 & Fe II[14F],55.14 & 0.22 \\
\hline 7280.8 & $\operatorname{HeI}(45), 81.35$ & abs \\
\hline 7463.0 & Fe II(73),62.6 & 0.06 \\
\hline 7494.6 & Fe II,95.6 & \\
\hline 7516.4 & Fe II(73), 15.3 & 0.04 \\
\hline 7712.3 & Fe II,11.4 & 0.18 \\
\hline 7771.9 & O I,72.0 & 0.16 \\
\hline 7865.5 & Fe II,66.5 & 0.15 \\
\hline 7876.4 & $\operatorname{Mg} \mathrm{II}(8), 77.1$ & \\
\hline 7895.9 & $\operatorname{Mg~II(8),96.4~}$ & \\
\hline 8423.1 & Fe II,23.9 & 0.07 \\
\hline 8445.9 & O I(4),46.0 & 3.83 \\
\hline 8468.0 & $\begin{array}{c}\text { H I Pa17,67.3 } \\
\text { Fe II,68.1 }\end{array}$ & \\
\hline 8498.1 & Ca II(2),98.0 & 3.88 \\
\hline 8594.3 & Н I Pa14,98.4 & \\
\hline 8616.9 & $\mathrm{Fe} \mathrm{II}[13 \mathrm{~F}], 17.0$ & \\
\hline 8661.0 & Ca II(2)62.1 & 4.41 \\
\hline 8664.9 & Н г Pa13,65.0 & \\
\hline 8722.0 & Fe II,22.4 & \\
\hline 8750.3 & Н I Pa12,50.5 & \\
\hline
\end{tabular}

and the [O I] multiplet at $\lambda \lambda 6300.2$ and 6363 are present, but quite weak. The photospheric features Si II $\lambda \lambda$ 6347,6371 


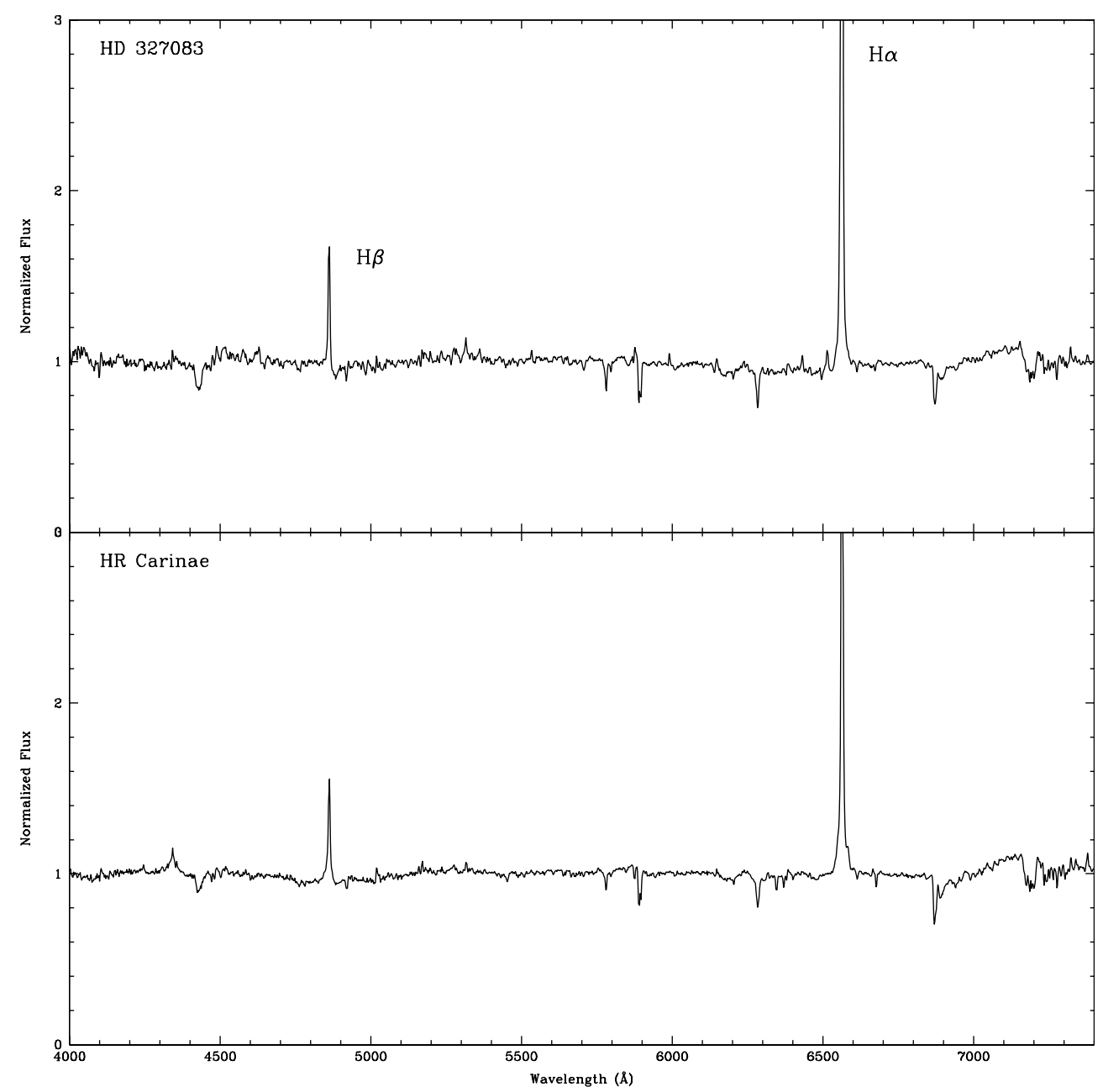

Fig. 1. Low resolution spectra of HD 327083 and HR Carinae obtained with the CASSEGRAIN spectrograph.

are present. According to Nota et al. (1997) this doublet can be used as a temperature indicator since it is present only at temperatures below than $19000 \mathrm{~K}$. We also observe the Mg II lines $\lambda \lambda 7877,7896(\mathrm{~m} 8)$ and $\lambda \lambda$ 8213, $8235(\mathrm{~m} 7)$ and the Ca II triplet $\lambda \lambda 8498-8662$ in emission.

The high resolution data allows a comparison of the spectra of HD 327083 and HR Carinae. Figure 2 presents a overview of selected regions of them while Figs. 3 and 4 show a set of iron and helium lines respectively. We can see that the Balmer lines in HD 327083 are also characterized by P-Cygni profiles. However the intensities and equivalent widths of their emission components are greater in HR Carinae. On the other hand the absorption components of $\mathrm{H} \beta, \mathrm{H} \gamma$ and $\mathrm{H} \delta$ are broader in HD 327083, indicating different wind densities. Almost all Fe II features in the two stars show P Cygni profiles, but the emission component is weaker in HD 327083. In addition, they are less numerous. In HR Carinae the absorption components of Fe II lines exhibit splitting features, not seen in HD 327083, that have been associated with the presence of two different shells in its outer atmosphere (Paper I). All He I lines of the two stars have in common the fact of being only in absorption. He I lines are useful as a temperature indicator and their weakness points in favour of a low value for the effective temperature (see Voors et al. 2000).

\section{The method of spectral analysis}

\subsection{The numerical code}

In order to derive physical parameters of massive stars with extended atmospheres from spectroscopic data one has to employ an atmospheric non-LTE model. Usual input parameters are stellar temperature $\left(T_{\star}\right)$, stellar luminosity $\left(L_{\star}\right)$, mass loss rate $(\dot{M})$, wind terminal velocity $\left(V_{\infty}\right)$, the power index of the wind velocity law $(\beta)$ and abundances $\left(A_{\mathrm{He}}\right.$, if only hydrogen and helium are considered). Each set of parameters is used as input data in a numerical code in order to produce synthetic profiles that are compared with the observed ones.

The code we use was developped by one of us (Machado 1998). It has been used in previous works (Machado et al. 2001, Paper I). The basic assumptions are: spherical symmetry, stationary and homogeneity. The density structure $\rho(r)$ is related to the mass loss rate and the velocity field via the equation of continuity. The velocity field is pre-specified in an adhoc way as a $\beta$-type law. The statistical equilibrium equations are solved using the escape probability method for calculating the source function while the transfer equation is solved using the "SEI" - Sobolev Exact Integration - method (Lamers et al. 1987). In the present work it is considered that the wind begins in the sonic point localized at radius $R_{\star}$. The stellar 

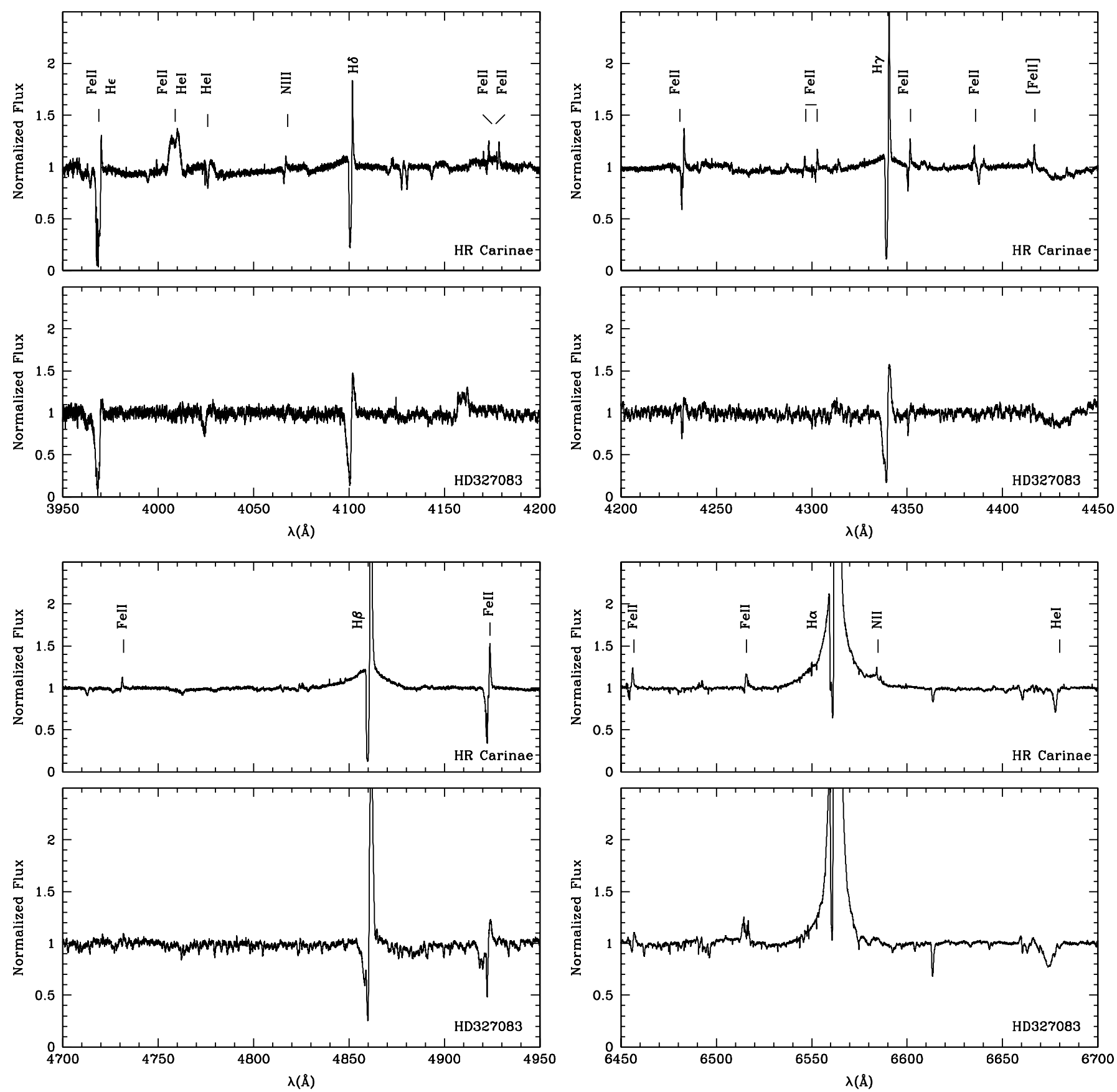

Fig. 2. Selected regions of the high-resolution spectra of HD 327083 and HR Carinae obtained with FEROS spectrograph.

temperature $T_{\star}$, the luminosity $L_{\star}$ and the radius $R_{\star}$ are related by the Stefan-Boltzmann law. Moreover, the model atmosphere consists of hydrogen and helium only. For hydrogen and single ionized helium we consider the lowest ten bound levels. For neutral helium we adopt the atomic model suggested by Almog \& Netzer (1989) that includes individual levels until $n \leq 4$ and combined levels with $5 \leq n \leq 10$. The determination of the population of each level considered in the atomic model is given by the solution of the equations of statistical equilibrium. The radiation and the statistical equations system are solved simultaneously through the wind that is divided in shells. A convergence criterium is applied in order to move from one shell to the next. The reader is referred to Paper I for further details on our code.

\subsection{Grid of models}

The fitting of observed lines can be a very difficult task not only due to the limitations of a specific code but also the number of "free" parameters. The approach employed by us is the following. First we constructed a primary grid of models (theoretical profiles) based on the results provided by theoretical evolutionary paths. In other words, the input parameters $L_{\star}, T_{\star}, \dot{M}$ and $A_{\mathrm{He}}$ are those provided as output data of the 

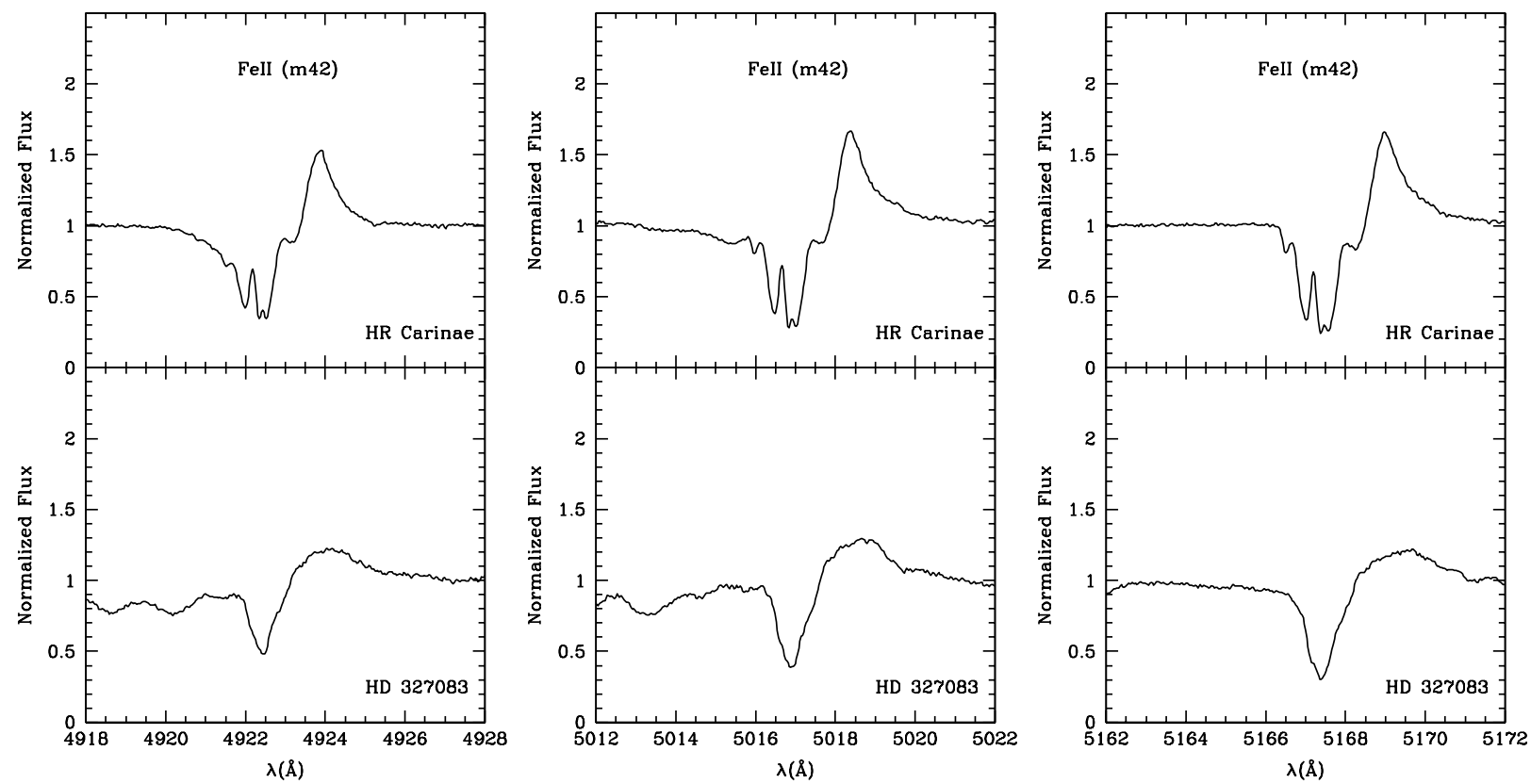

Fig. 3. High-resolution data of Fe II lines (m42) of HD 327083 and HR Carinae.
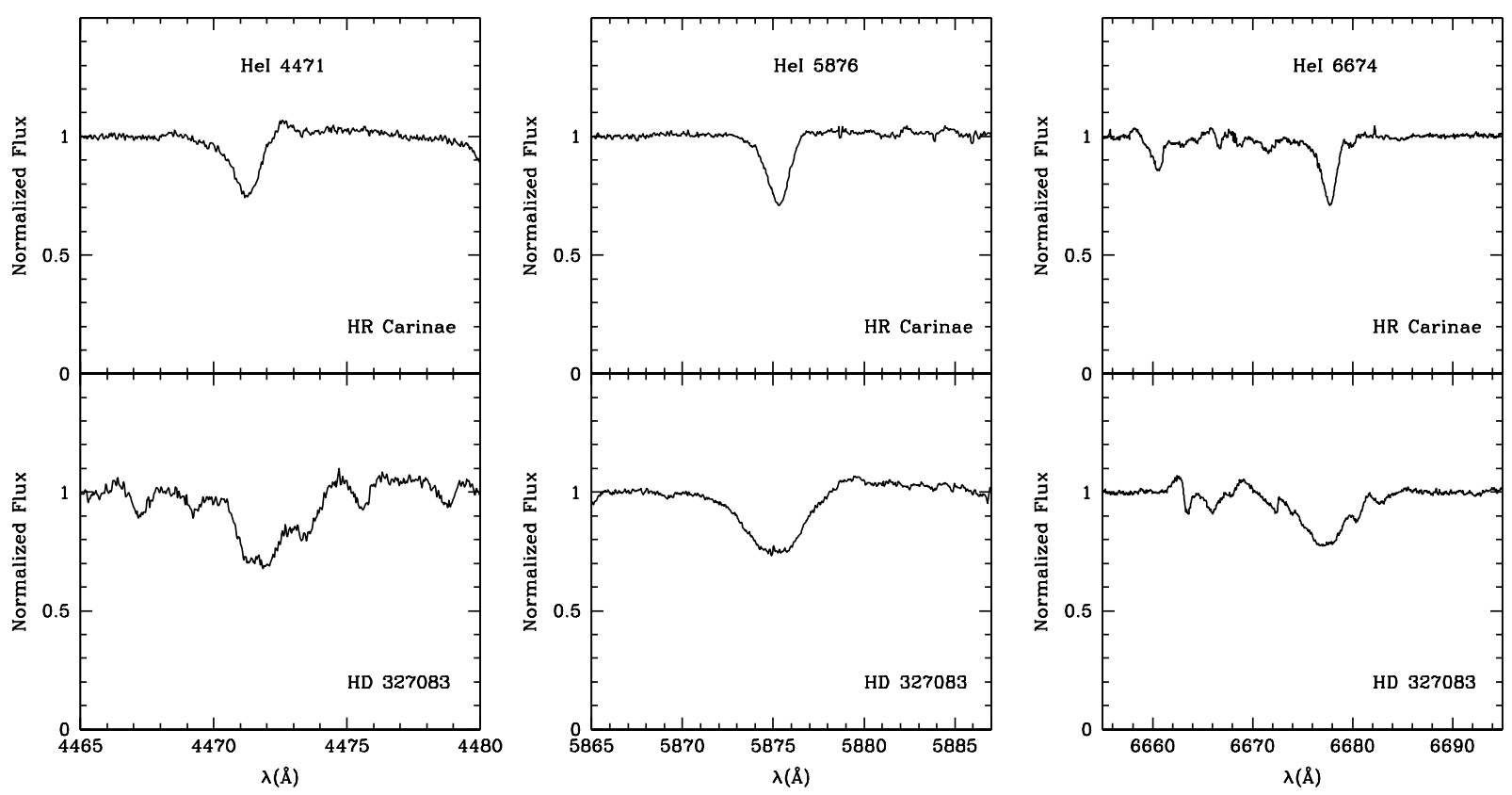

Fig. 4. High-resolution data of He I lines of HD 327083 and HR Carinae.

evolutive models. We chose the tracks of Schaller et al. (1992) since they are available on the WEB in the range of initial masses from $M_{\mathrm{ZAMS}}=25 M_{\odot}$ to $120 M_{\odot}$. These tracks are computed for non-rotating, solar metalicity stars. We are aware that recent computations considering high rotation and low metalicities may lead to important changes. Our calculations were performed for all points until the end of hydrogen in the surface. We obtained theoretical profiles of Balmer lines and He I $\lambda 5876$ and $\lambda 6671$. The terminal velocity of the wind was taken from the observations.

In addition to the grid of models we have investigated the influence of each parameter in the line profiles. The details of these studies are in Nascimento (2003). We will report here two results that are very useful in the present work. The first concerns the behavior of $\mathrm{He} \mathrm{I}$ lines according to the variation of temperature. If we fix a physically reasonable set of parameters and vary only the temperature, we note that for values smaller than a critical one (that in our case is about $13000 \mathrm{~K}$ ) the emission component of He I lines disappear (see Fig. 5). This is a very significant result for the purposes of the present work since in our high-resolution, good signal to noise ratio spectra of HD 327083 no emission at all is seen in He I lines. The second result refers to stellar luminosity. If we choose any model and increase only the luminosity, keeping fixed the other parameters, we obtain that the intensities of Balmer lines decrease. This can be understood in terms of the continuity law. 

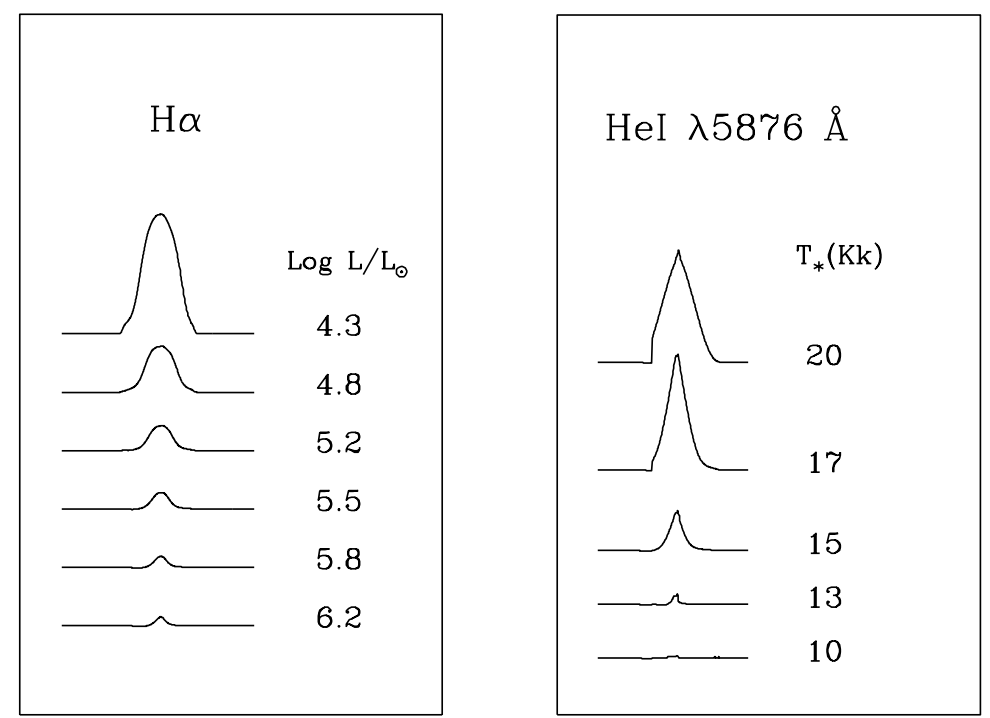

Fig. 5. Behavior of $\mathrm{H} \alpha$ and $\mathrm{He} \mathrm{I} \lambda 5876$ lines under variations in luminosity and temperature respectively. The other input parameters are $\dot{M}=3.0 \times 10^{-5} M_{\odot} \mathrm{yr}^{-1}$ and $A_{\mathrm{He}}=0.4$ (by number). In this figure, wavelengths in $\AA$ are given in abscissa and the ordinate corresponds to continuum flux units.

If we increase the luminosity with the temperature fixed, the stellar radius increases too. Consequently the density decreases and the Balmer lines will also decrease. Most importantly, this effect is quite strong (see again Fig. 5). These two results and our first preliminary analysis of HD 327083 (Machado et al. 2001) will be invoked to help us to estimate the physical parameters of this star.

\section{Results: The stellar parameters of HD $\mathbf{3 2 7 0 8 3}$}

In our previous analysis of HD 327083 (Machado et al. 2001), we showed that $\mathrm{H} \alpha$ and $\mathrm{H} \beta$ line profiles could be fairly well reproduced with two different set of parameters. One set (called Model 1 in that work) had $T_{\star}=19000 \mathrm{~K}$ while the other one (Model 2) had $T_{\star}=9000 \mathrm{~K}$. We remind the reader that helium lines have not been considered in that analysis due to the lack of reliable data. The definitive absence of emission in the He I lines in our new high resolution spectra strongly favours the lower temperature $\left(T_{\star} \leq 13000 \mathrm{~K}\right.$, according to our computations cited in Sect. 3.2). Then, we took the parameters of Model $2\left(\dot{M}=1.0 \times 10^{-4} M_{\odot} \mathrm{yr}^{-1}, T_{\star}=9000 \mathrm{~K}\right.$, $\left.L_{\star}=9.5 \times 10^{5} L_{\odot}, A_{\mathrm{He}}=0.11\right)$, that correspond to a point not far from the $M_{\text {ZAMS }}=60 M_{\odot}$ evolutionary path, as a starting point in order to obtain the best fit of Balmer lines $(\mathrm{H} \alpha, \mathrm{H} \beta$, $\mathrm{H} \gamma$ and $\mathrm{H} \delta$ ). The terminal velocity was taken from the spectra. We have used the lines $\mathrm{H} \delta$, that has an absorption component free of blends or structures, and He I $\lambda 3888$ to measure $V_{\infty} \sim 400 \mathrm{~km} \mathrm{~s}^{-1}$. As these lines should be formed inner in the wind, we explored slightly greater velocities and reached to the conclusion that the best value to reproduce the lines is $\sim 450 \mathrm{~km} \mathrm{~s}^{-1}$.

The fits of the lines are mainly determined by an adequate combination of stellar temperature, luminosity and wind density. The density is given by mass loss rate, terminal velocity and stellar radius (or luminosity, since they are related once a temperature value is given), via the equation of mass conservation. As in our approach the input parameters of the numerical code are based on output parameters of evolutionary models, we worked with the stellar luminosity rather than the radius. The behavior of the theoretical profiles of the He I $\lambda 5876$ and $\lambda 6671$ lines lead to a temperature range $11000-12000 \mathrm{~K}$. In addition, we have used the influence of luminosity on the Balmer lines. We confirmed that a decreasing of luminosity implies in an increasing of emission intensity specially in the cases of $\mathrm{H} \alpha$ and $\mathrm{H} \beta$. From this, it was possible to determine a value $L \sim 10^{6} L_{\odot}$. Of course, the mass loss rate also plays a role in the fitting of the profiles. However, the possible effects of the variation of mass loss rate in the Balmer lines can be somewhat controled by helium abundance.

Our fits of Balmer lines for HD 327083 are shown in Fig. 6. The corresponding stellar and wind parameters are in Table 2. From the figure we can see that the emission component of the P Cygni profiles could be quite well reproduced. However, the fits of the blue-shifted absorptions are rather poor: the model is not able to produce structures like those seen more clearly in $\mathrm{H} \alpha$ anf $\mathrm{H} \beta$ and it underestimates $\mathrm{H} \gamma$ and $\mathrm{H} \delta$ absorption strengths. The problems are probably related to limitations of the model, mostly homogeneity and spherical expansion. In spite of these difficulties, it is quite sure that we need a high mass loss rate $\left(\dot{M}=8.0 \times 10^{-5} M_{\odot} \mathrm{yr}^{-1}\right)$ combined with an increased helium abundance $\left(A_{\mathrm{He}}=0.2\right)$ in order to obtain the strengths of the Balmer lines. The temperature $T_{\star}=11500 \mathrm{~K}$ is above all linked to the absence of He I emissions, while a luminosity $L_{\star}=10^{6} L_{\odot}$ is necessary to allow that the emission of Balmer lines can be reproduced.

Table 2 also lists the parameters obtained for HR Carinae in Paper I. It can be seen that some parameters of HD 327083 are similar to those of HR Carinae but others are not. This result is not surprising given the similarities (Fig. 1) and differences (Figs. 2, 3) between their spectra. The most important difference is the stellar luminosity, that is much greater for HD 327083; this happens essentially because its emission 
HD 327083
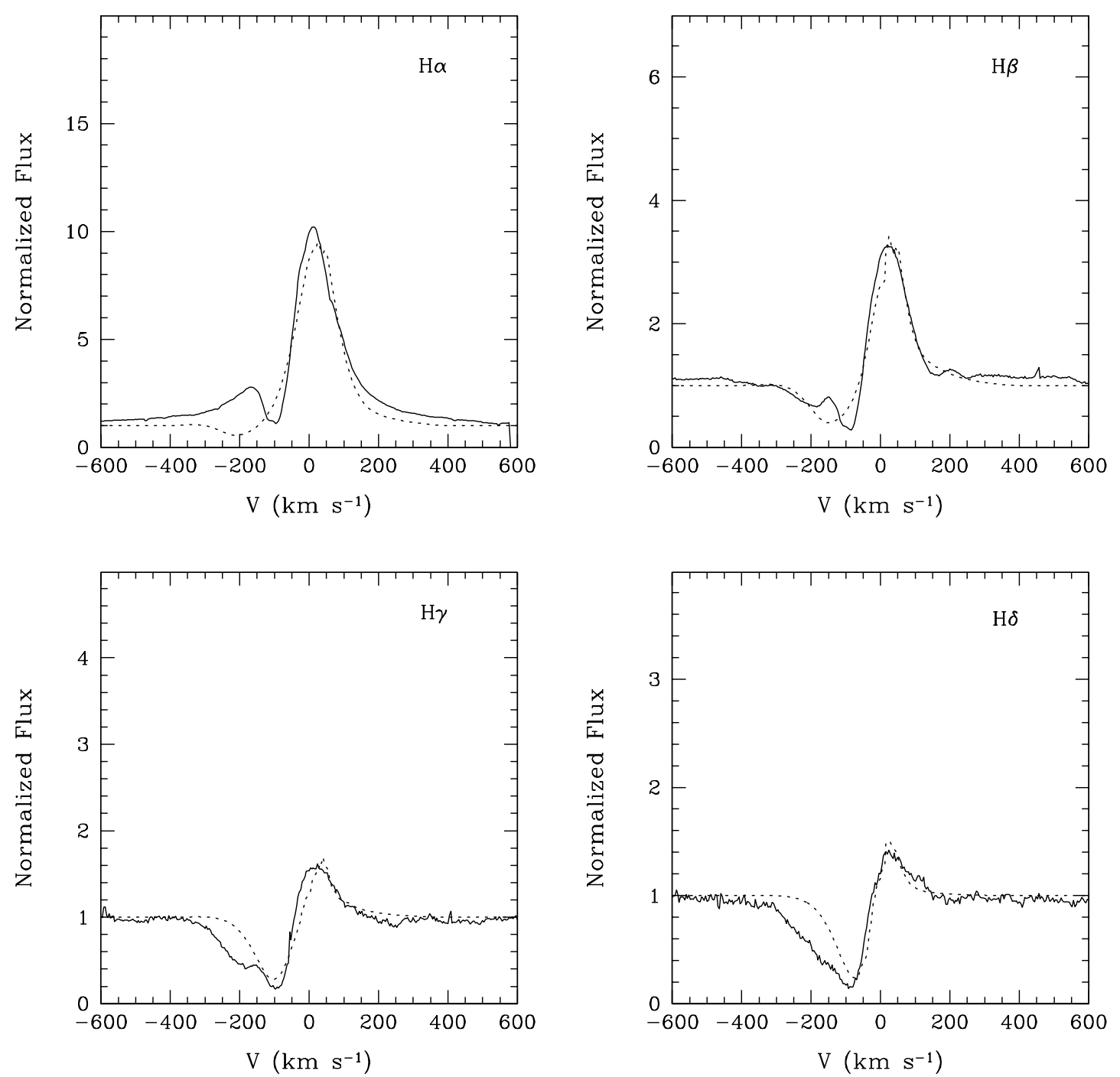

Fig. 6. Observational data (full lines) and best theoretical fittings (dashed lines) for $\mathrm{H} \alpha, \mathrm{H} \beta, \mathrm{H} \gamma$ and $\mathrm{H} \delta$ profiles.

Table 2. Stellar parameters derived for HD 327083 and HR Carinae.

\begin{tabular}{lccccc}
\hline \hline Object & $\log \left(L_{\star} / L_{\odot}\right)$ & $T_{\star}(\mathrm{K})$ & $\dot{M}$ & $V_{\infty}$ & $A_{\mathrm{He}}$ \\
\hline HD 327083 & $1.0 \mathrm{E} 06$ & 11500 & $8.0 \mathrm{E}-05$ & 450 & 0.2 \\
HR Carinae & $5.0 \mathrm{E} 05$ & 10000 & $6.8 \mathrm{E}-05$ & 400 & 0.4 \\
\hline
\end{tabular}

intensities are weaker. In the next section we will discuss the parameters derived and their implications.

\section{Discussion: Physical parameters and evolutionary status of HD 327083}

Let us begin by commenting on the luminosity, since the value we have estimated is quite high. First it should be noted that there are almost no determinations of luminosity for HD 327083 in the literature. As far as we know the sole exception may be found in Lopes et al. (1992). From their values of distance and bolometric magnitude it is obtained $\log \left(L / L_{\odot}\right)=6.26$. This even higher value was adopted by
Araújo \& Freitas Pacheco (1994) and Sheikina et al. (2000) but these last authors worked with $\log T_{\text {eff }} \geq 4.2$. Moreover, Lopes et al.'s estimate of distance $(d=5.0 \mathrm{kpc})$ should be taken with great caution since it was based on the statistical relation with the equivalenth width of interstellar $\mathrm{Na}$ I $D_{1}$ and $D_{2}$ lines (Allen 1973). On the basis of the same method our data indicates a slightly smaller distance $(d=4.8 \mathrm{kpc})$. In fact, obtaining reliable distances to peculiar galactic stars is a difficult task. In some cases it is possible to compare the systemic velocity of interstellar lines to the galactic rotation curve. This method is not appropriate to HD 327083 due to its galactic location. We also tried to estimate its reddening using the diffuse interstellar bands, following the results described by Herbig (1995). From the equivalent widths of the $\lambda 5795$ and $\lambda 6614$ bands relative to those in HD 183413 we have derived $E_{B-V}=1.3$. Unfortunately, this method often leads to inaccurate values (see, for instance, Hillier et al. 1998). For the sake of completeness we cite that Lopes et al. (1992) have determined $E_{B-V}=1.8$. On the other hand, we emphasyze again that the luminosity value was quite well constrained in our model 


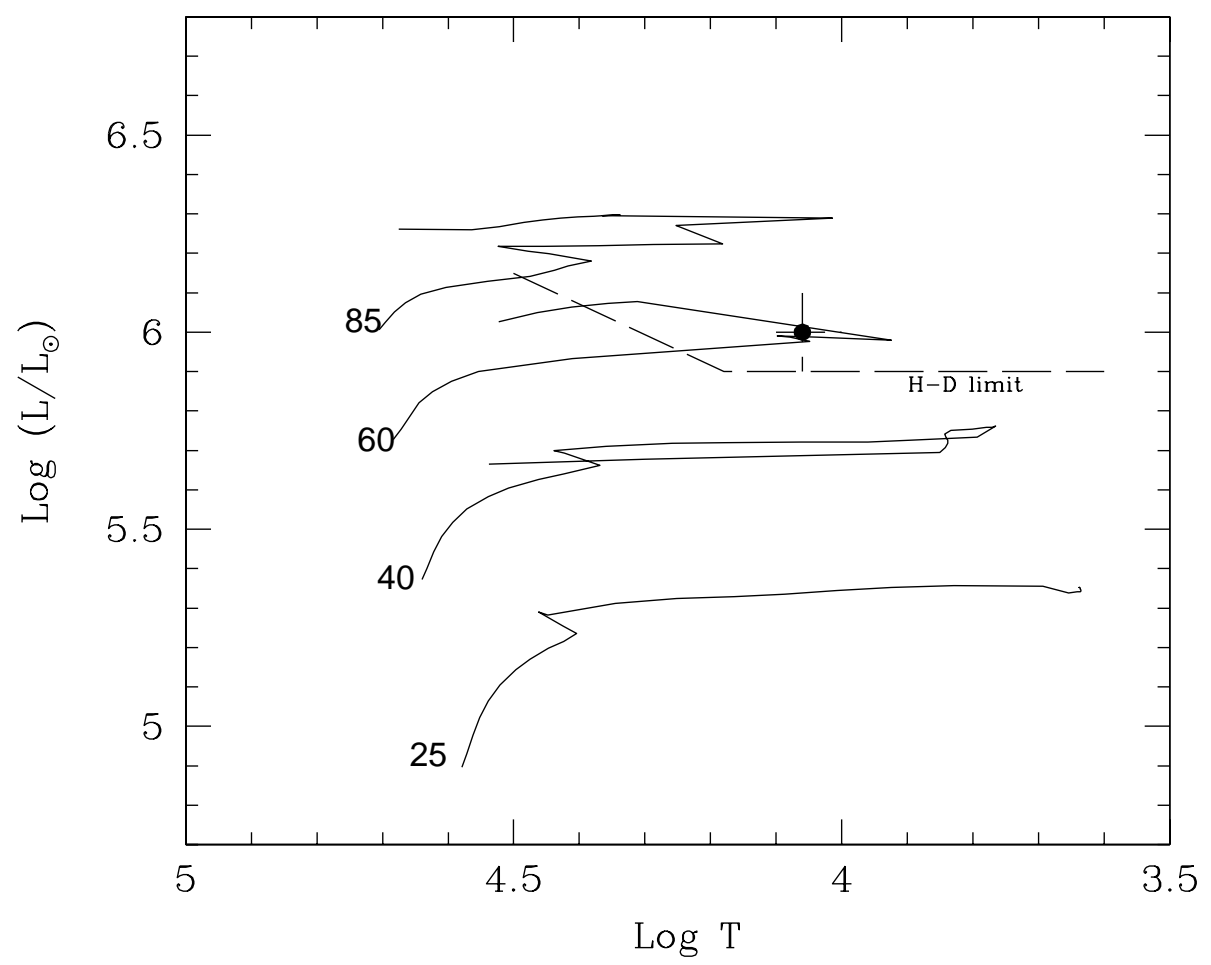

Fig. 7. Theorethical evolutionary tracks of $25 M_{\odot}, 40 M_{\odot}, 60 M_{\odot}, 85 M_{\odot}$ initial main-sequence mass (full lines) and the empirical Hummphreys-Davidson limit (dashed lines). The full circle locates HD 327083 according to our result.

by the fitting of the emission components of the Balmer lines. Thus we can safely conclude that our value is a fairly good first quantitative determination.

We will discuss now the stellar temperature. Previous works of different authors have only suggested spectral types: B6 (Lopes et al. 1992), B1.5 (Thé et al. 1994), B3-6 (Sheikina et al. 2000). These types correspond to a temperature range ( 13000-20000 K) above our estimate. However, these attempts have rest on crude assumptions and we see no reasons to discard our result based on them. The question that arises is that the high luminosity $\left(L / L_{\odot}=10^{6.0}\right)$ combined with the low temperature $\left(T_{\star}=11500 \mathrm{~K}\right)$ places HD 327083 above the Humphreys-Davidson's (1979) limit. This empirical limit in the HR diagram is characterized by decreasing luminosity with decreasing temperature for the hottest stars and an upper limit to the luminosities near $L \approx 10^{5.9}-10^{6.0} L_{\odot}$ for stars cooler than $\approx 15000 \mathrm{~K}$. Later, Humphreys (1993) cited three type-A hypergiants that rest in more or less the same region of the HR diagram as HD 327083 does. Their temperatures are likely to be in the domain $9000-11500 \mathrm{~K}$ and their luminosities in the range $10^{5.9}-10^{6.1} L_{\odot}$. One of these stars is Cyg OB2 \# 12 that according to Massey \& Thompson (1991) may be an incipient LBV that has undergone a previous episode of mass loss. From the theoretical point of view, evolutionary paths with $M_{\text {ZAMS }} \sim 60-85 M_{\odot}$ do predict the possibility of objects with such parameters. This is true not only for the models we have adopted (Schaller et al. 1992) but also for models that assume enhanced mass loss rates (Meynet et al. 1994). The points $\left(L_{\star}, T_{\star}\right)$ of a given evolutionary track that lie in the region above the Humphreys-Davidson's limit in the HR diagram are related to phases of short duration. For instance, in the $M_{\mathrm{ZAMS}}=60 M_{\odot}, \mathrm{Z}=0.020$ path, this phase corresponds to less than $5 \%$ of the total.

In Fig. 7 we see that the position where HD 327083 is placed corresponds to the reddest points of Schaller et al. (1992) track of $M_{\text {ZAMS }}=60 M_{\odot}$. In this region the mass loss rate is high, independent of whether the object is moving redward or blueward. The unique parameter that can distinguish among these two possibilities is the chemical abundance. The helium abundance derived by us indicates that HD 327083 has crossed the HR diagram redward and is starting to move towards the hotter region of the diagram. If our results are confirmed, HD 327083 may be a rare example of an object that is in a short-lived evolved stage of a highly massive star.

\section{Summary and conclusions}

HD 327083 has been cited many times in the literature as a peculiar emission-line star with strong mass loss. Different evolutive stages may be associated to such objects. From pre-main sequence stars of intermediate masses to evolved supergiant massive stars. HD 327083 was listed as an early-type highluminosity star by McGregor et al. (1988), Lopes et al. (1992) and Miroshnichenko et al. (1995). On the other hand it was included by Thé et al. (1994) in their catalogue of Herbig AeBe stars.

The Balmer serie lines and most of the permitted iron lines of HD 327083 show P Cygni profiles. However, its spectrum can be considered poor in iron when compared to other emission-line supergiants. We have also identified some lines of $\mathrm{N}$ II and $\mathrm{OI}$ that show evidence of $\mathrm{CNO}$ processing. In addition, McGregor et al. (1988) reported the 
detection of CNO that should have originated in circumstellar shells. According to them, these shells can be formed in a supergiant phase. The overall spectral morphology of HD 327083, including the $\mathrm{CO}$ emission, resembles that of the low-luminosity LBV HR Carinae but important differences may be easily found in a careful inspection.

In the present work we showed that HD 327083 is losing mass at an intense rate and has an enhanced helium abundance. Taking into account the stellar temperature and luminosity, we conclude that this object is in an evolved phase of an evolutive track of a star with an initial mass of the order of sixty solar masses, coming back to the blue side of the HR Diagram. An object in this phase should have remnant mass loss envelopes that could be the source of the $\mathrm{CO}$ emission detected by McGregor et al. (1988). In addition, other objects are supposed to be in the same region of the HR diagram. Cyg OB2 \# 2 is one of them and it may be an incipient LBV (Massey \& Thompson 1991). This evidence indicate that the evolutionary phase of HD 327083 is related to the LBV phase of a highly massive star.

Acknowledgements. M. Machado thanks CNPq (200238/0600-1) for financial support.

\section{References}

Allen, C. W. 1973, Astrophysical Quantities (London: The Athlone Press)

Almog, Y., \& Netzer, H. 1989, MNRAS, 238, 51

Araújo, F. X., \& Freitas Pacheco, J. A. 1994, Ap\&SS, 219, 267

Bernat, A. P., \& Lambert, D. L. 1978, PASP, 90, 520

Carlson, E. D., \& Henize, K. G. 1979, Vist. Astron., 23, 213

Davidson, K. 1987, ApJ, 317, 760

Fernandes, M. B., de Araujo, F. X., Pereira, C. B., \& Landaberry, S. J. C. 2001, ApJS, 136, 747

Garcia-Lario, P., Riera, A., \& Manchado, A. 1998, ApJ, 526, 854

Herbig, G. H. 1995, ARA\&A, 33, 19

Hillier, D. J. 1987, ApJS, 63, 947

Hillier, D. J. 1990, A\&A, 231, 116

Hillier, D. J., Crowther, P. A., Najarro, F., \& Fullerton, A. W. 1998, A\&A, 340, 483

Humphreys, R. M. 1993, in Massive Stars: Their Lives in the Interstellar Medium, ed. J. P. Cassinellli, \& E. B. Churchwell, ASP Conf. Ser., 35, 179,

Humphreys, R. M., \& Davidson, K. 1979, ApJ, 232, 409

Kaufer, A., Stahl, S., Tubbesing, S., et al. 1999, Messenger, 95, 8

Lamers, H. J. G. L. M., Cerruti-Sola, M., \& Perinoto, M. 1987, ApJ, 314,762

Landaberry, S. J. C., Pereira, C. B., \& de Araújo, F. X. 2001, A\&A, 376,917
Langer, N., Hamann, W.-R., Lennon, M., et al. 1994, A\&A, 290, 819

Lopes, D. F., Damineli Neto, A., \& de Freitas Pacheco, J. A. 1992, A\&A, 261, 482

Machado, M. 1998, in Determinação de parâmetros estelares de objetos massivos: aplicação as estrelas WCs e LBVs, Ph.D. Thesis, Observatório Nacional, Brazil

Machado, M., de Araújo, F. X., \& Lorenz-Martins, S. 2001, A\&A, 368, L29

Machado, M., de Araújo, F. X., Pereira, C. B., \& Fernandes, M. B. 2002, A\&A, 387, 151 (Paper I)

Maeder, A., \& Meynet, G. 1987, A\&A, 182, 243

Maeder, A., \& Meynet, G. 2000, A\&A, 361, 101

Massey, P., \& Thompson, A. B. 1991, AJ, 101, 1408

McGregor, P. J., Hyland, A. R., \& Hillier, D. J. 1988, ApJ, 324, 1071

Meynet, G., Maeder, A., Schaller, G., Schaerer, D., \& Charbonnel, C. 1994, A\&AS, 103, 97

Miroshnichenko, A. S., Bergner, Yu. K., Mukanov, D. B., \& Shejkina, T. A. 1995 , Ap\&SS, 224, 519

Moore, C. E. 1945, A Multiplet Table of Astrophysical Interest, Part I, Table of Multiplets, revised ed. (Princeton: Princeton Univ. Obs.)

Morris, P. W., Eenens, P. R. J., \& Blum, R. D. 1996, ApJ, 470, 597

Nascimento, C. 2003, in Uma Grade de Perfis Teóricos para Modelos Evolutivos, MSc. Thesis, Observatorio Nacional, Brazil

Najarro, F., Hillier, D. J., \& Stahl, O. 1997, A\&A, 326, 1117

Nota, A., Pasquali, A., Drissen, L., et al. 1996, ApJS, 102, 383

Nota, A., Smith, L., Pasquali, A., Clampin, M., \& Stroud, M. 1997, ApJ, 486, 338

Pasquali, A., Schumutz, W., Nota, A., \& Origlia, L. 1997, A\&A, 327, 265

Schaller, G., Schaerer, D., Meynet, G., \& Maeder, A. 1992, A\&AS, 96, 269

Schmutz, W., Leitherer, C., Hubeny, I., et al. 1991, ApJ, 372, 664

Schmutz, W. 1997, in Luminous Blue Variables: Massive stars in transition, ed. A. Nota, \& H. J. G. L. M. Lamers, ASP Conf. Ser., 120, 143

Schulte-Ladbeck, R. E., Leitherer, C., Clayton, G. C., et al. 1993, ApJ, 407, 723

Sheikina, A., Miroshnichenko, A. S., \& Corporon, P. 2000, in The Be phenomenon in Early-Type Stars, IAU Colloq. 175, ed. M. A. Smith, H. F. Henrichs, \& J. Fabregat, ASP Conf. Ser., 214, 494

Smith, L. J., Crowther, P. A., \& Prinja, R. K. 1994, A\&A, 281, 833

Stahl, O. 1987, A\&A, 182, 229

Stahl, O., Wolf, B., Klare, G., et al. 1983, A\&A, 127, 49

Stahl, O., Wolf, B., \& Zickgraf, F. J. 1996, A\&A, 314, 131

Stahl, O., Jankovics, I., Kovács, J., et al. 2001, A\&A, 375, 54

Thé, P. S., de Winter, D., \& Pèrez, M. R. 1994, A\&AS, 104, 315

Valenti, J. A., Johns-Krull, C. M., \& Linsky, J. L. 2000, ApJS, 129, 399

Voors, R. H. M., Geballe, T. R., Waters, L. B. F. M., Najarro, F., \& Lamers, H. J. G. L. M. 2000, A\&A, 362, 236

Walborn, N. R. 1982, ApJ, 256, 45 